\title{
PERFORMANCE OF THE CESR HIGH LUMINOSITY INTERACTION REGION
}

\author{
S. Henderson*, Laboratory of Nuclear Studies, Cornell University, Ithaca, NY $14853^{\dagger}$
}

\begin{abstract}
In November 1995, the Cornell Electron Storage Ring (CESR) began Phase II operations. The CESR/CLEO Phase II upgrade involved the installation of a silicon vertex detector for the CLEO experiment, and an interaction region optimized for a small-radius $(2 \mathrm{~cm})$ beampipe and bunch-train collisions at total beam currents of $600 \mathrm{~mA}$. The operating experience of the CESR Phase II Interaction Region is reviewed.
\end{abstract}

\section{INTRODUCTION}

The Cornell Electron Storage Ring (CESR) began Phase II Operation in November 1995 following the installation of the Phase II Interaction Region (IR). The Phase II IR was optimized for bunch-train collisions at a finite crossing angle to provide a design luminosity of $6 \times 10^{32} \mathrm{~cm}^{-2} \mathrm{sec}^{-1}$. As part of the upgrade project, the CLEO Experiment installed a silicon vertex detector (SVX) [1] around a smallradius beampipe. The detector background shielding system was designed to minimize backgrounds and radiation dose to the silicon detector and associated electronics in the presence of crossing angle collisions at design currents of $300 \mathrm{~mA} e^{+}+300 \mathrm{~mA} e^{-}$. After an initial commissioning period in which the vacuum system was beam processed, CESR beam currents increased, and the luminosity followed. CESR presently operates [2] at peak colliding beam currents of $\sim 340 \mathrm{~mA}$ and a peak luminosity of $4.1 \times 10^{32}$ $\mathrm{cm}^{-2} \mathrm{sec}^{-1}$. CESR has delivered $3.9 \mathrm{fb}^{-1}$ since the Phase II start-up.

\section{CESR PHASE II}

CESR Phase II exploits an idea put forth by Meller [3] to increase the number of bunches in each beam by colliding the beams at a small horizontal angle $\left(\theta_{c} \simeq \pm 2 \mathrm{mrad}\right)$. This crossing angle allows "trains" of closely spaced bunches (with minimum bunch spacing of 14ns) to be collided; the angle generates adequate separation of the beams at the nearby parasitic crossings in the IR. The beams follow "pretzel" orbits in the arcs, and are thus separated at each parasitic crossing.

The presence of the crossing angle has several important consequences for the IR design. First, the off-axis beams in the IR quadrupoles generate synchrotron radiation (SR) which must be properly masked, and this masking must in turn be adequately cooled. Second, the crossing angle displaces the beams in the horizontally focussing IR

\footnotetext{
* Representing the CESR Operations Group

$\dagger$ Work supported by the National Science Foundation
}

\begin{tabular}{|l|c|}
\hline$E$ & $5.3 \mathrm{GeV}$ \\
$\mathcal{L}$ & $6.0 \times 10^{32} \mathrm{~cm}^{-2} \mathrm{sec}^{-1}$ \\
$\xi_{v}$ & 0.03 \\
$C$ & $768 \mathrm{~m}$ \\
$N_{b}$ & 27 per beam \\
$I$ & $300 \mathrm{~mA} \mathrm{per} \mathrm{beam}$ \\
$\theta_{c}$ & $2.5 \mathrm{mrad}$ \\
$\beta_{v}^{*}$ & $1.9 \mathrm{~cm}$ \\
$\beta_{h}^{*}$ & $1.2 \mathrm{~m}$ \\
$\eta^{*}$ & $0 \mathrm{~m}$ \\
$\sigma_{l}$ & $1.8 \mathrm{~cm}$ \\
$\epsilon_{h}$ & $0.21 \mathrm{~mm} \mathrm{mrad}$ \\
$Q_{x}$ & 10.53 \\
$Q_{y}$ & 9.60 \\
\hline
\end{tabular}

Table 1: CESR Phase II Design Parameters

quadrupole, thereby reducing the aperture. Adequate space must therefore be provided for the injected bunch oscillations.

The CESR Phase II design parameters are shown in Table 1 . The design calls for 9 trains per beam and 3 bunches per train for a total beam current of $600 \mathrm{~mA}$. At these currents the expected luminosity is $6.0 \times 10^{32} \mathrm{~cm}^{-2} \mathrm{sec}^{-1}$.

\section{PHASE II INTERACTION REGION}

The IR design had to accomodate competing requirements from the accelerator and the detector. To take full advantage of the precision tracking capability of the SVX, it was important to allow the first tracking layer to be as close to the interaction point (IP) as possible while still maintaining acceptable background levels in the face of a 3-fold increase in beam currents. The SVX readout electronics, the most radiation sensitive component in the IR, were measured [4] to fail at 25-35 krad accumulated dose when powered. (The lifetime dose when unpowered is $\sim 80-100 \mathrm{krad}$. The detector background design goals were i) SVX layer 1 occupancy $<1 \%$ hits $/$ strip $/ \mu$ sec and ii) SVX dose (when powered) $\leq 20 \mathrm{krad}$ in 3 years. In addition, the SVX temperature stability requirements are $\pm 5^{\circ} \mathrm{C}$.

Meanwhile, the accelerator design requires minimization of HOM-generation and adequate IR aperture for injected bunch oscillations into crossing angle orbits.

The IR design was based largely on simulation of detector backgrounds and radiation dose (described below). We determined that a beampipe radius of $2 \mathrm{~cm}$ gave acceptable backgrounds and set the first layer of Si at $r=2.35$ 


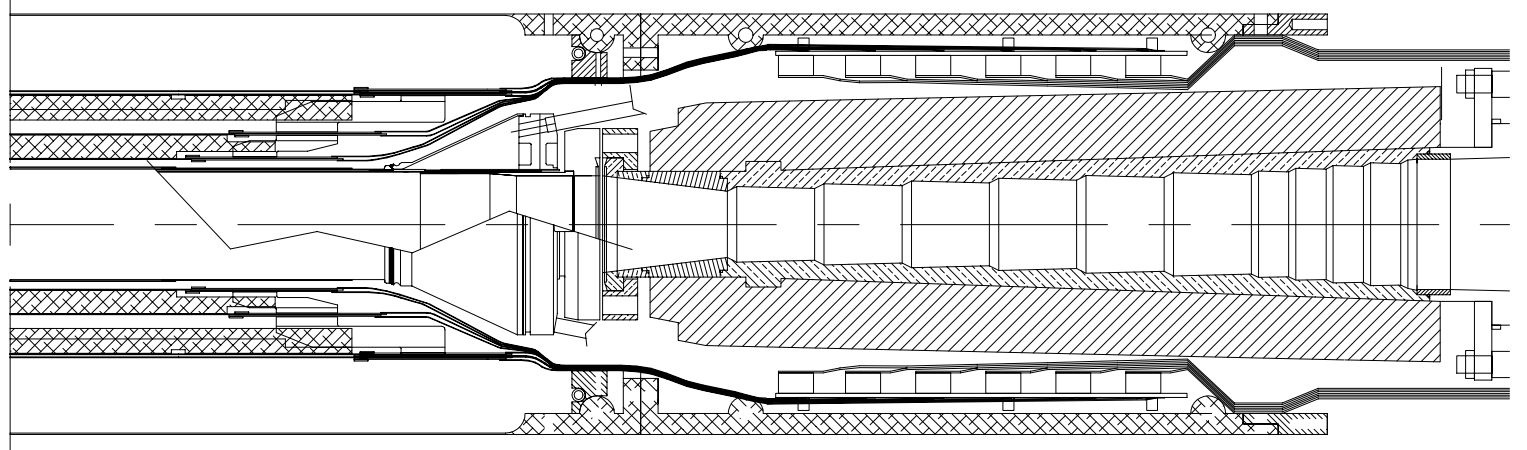

Figure 1: Interaction Region layout from the IP to $52 \mathrm{~cm}$. Starting at the IP and moving radially outward are the Be beampipe (extending to $21 \mathrm{~cm}$ ), three layers of Si tracking and the carbon fiber support tube. Starting in the middle of the figure and moving outward is the stepped copper SR mask $(21-50 \mathrm{~cm})$, tungsten shielding, SVX receiver electronics and carbon fiber housing.

$\mathrm{cm}$. The Phase II interaction region upgrade consisted of installing the following components: i) a $2 \mathrm{~cm}$ radius beryllium beampipe, ii) the CLEO SVX, iii) the detector background shielding system, iv) IR instrumentation, and v) new vacuum system components within $\pm 12 \mathrm{~m}$ of the IP. In addition, the final focus quadrupoles were modified. The Phase II IR from the IP to $52 \mathrm{~cm}$ is shown in Figure 1.

\subsection{Interaction Region Optics}

A rearrangement (from Phase I) of final focus quadrupoles made for more favorable IR optics [2]. The vertically focussing permanent magnet quadrupole was lengthened, allowing the horizontally focussing IR quadrupole to be moved closer to the interaction point. This reduces the maximum horizontal $\beta$ in the IR to $\sim 60 \mathrm{~m}$ (an improvement over the Phase I optics which had $\hat{\beta}_{h} \simeq 100 \mathrm{~m}$ ). Injection performance is improved since the maximum excursion of the injected bunch is reduced at this point. In addition, the physical aperture in the electromagnetic IR quads (Q1 and Q2) was increased by enlarging the pole tip radii, providing an additional $\sim 2 \mathrm{~cm}$ of horizontal aperture. These two features improve CESR injection and reduce detector backgrounds during injection.

\subsection{Central Beampipe}

The central beampipe is a $2 \mathrm{~cm}$ outer radius double-walled water cooled beryllium beampipe [5]. The beampipe, manufactured by Brush-Wellman Inc. ${ }^{1}$, was assembled from two Be tubes; the inner tube had ribbed supports. A 20 mil gap between Be tubes was provided for coolant flow. Manifolds at each end provide inlets and outlets to four separate cooling channels. Water was selected as the coolant because of its excellent thermal conductivity, capable of dissipating $400 \mathrm{~W}$ with only $5^{\circ} \mathrm{C}$ temperature rise.

The inner surface of the Be beampipe was coated with $10 \mu \mathrm{m}$ gold for the absorption of scattered synchrotron ra-

\footnotetext{
${ }^{1}$ Brush Wellman ADC, 34325 Ardenwood Boulevard, Fremont, CA ${ }_{94555}{ }^{1} \mathrm{Br}$
}

diation. The gold was deposited with a cylindrical magnetron sputtering technique [6]. The total beampipe thickness is $0.6 \% X_{0}$, half due to the gold coating and half due to $\mathrm{Be} / \mathrm{H}_{2} \mathrm{O}$.

Since corrosion of bare $\mathrm{Be}$ by ions in water is problematic, a layer of epoxy was applied to all bare Be surfaces by the manufacturer following the gold coating process.

\subsection{Detector Background Shielding}

The detector shielding system [7] minimizes beamgenerated backgrounds arising from two sources: beamgas interactions (Coulomb scattering and Bremsstrahlung) and scattered synchrotron radiation. Bremsstrahlung interactions result in beam particles which have lost a portion of their energy and which become over bent in dipole magnets and over focused in quadrupoles. Such "lost particles" may be directed into the experimental detector by the IR quadrupoles. In addition, Bremsstrahlung photons are generated and may strike near the IP. Coulomb interactions result in full energy beam particles which have large oscillation amplitudes and may strike the vacuum chamber near the IP.

Synchrotron radiation, generated in the final bend before the IR straight and in the IR quadrupoles due to the crossing angle trajectory, is directed toward the interaction point, and if not intercepted would strike the Be beampipe directly, leading to unacceptably large $x$-ray fluxes in the inner detectors.

The detector shielding consists of two parts. The detector is shielded from lost beam particles with massive $\mathrm{W}$ shielding, chosen for its short radiation length. The detector is shielded from synchrotron radiation by a stepped copper mask with innermost tip radius $r=1.2 \mathrm{~cm}$. The mask is profiled in such a way that SR photons strike only the "tips" of the mask, and no flux is received on other surfaces. $X$-rays striking near the tip may forward scatter into the beampipe and detector, generating background (termed "tipscattering" in this paper). Additionally, $x$-rays which 
pass over the innermost tip may strike the back surface of the mask. These photons may interact, producing fluorescent $x$-rays which strike the beampipe. (This process is termed "backscattering").

The choice of scattering materials and beampipe coating material was based on $x$-ray scattering Monte-Carlo detector background simulations. In particular, the gold beampipe coating material was chosen because its $\mathrm{L}$ absorption edge was found to be a good match to the scattered $x$-ray flux.

To monitor radiation levels in the IR, the beampipe has been instrumented with 12 PIN diode detectors at each end, sensitive to charged particles, as well as $x$-rays. The monitoring system is continuously read out and logged, and is used for background tuning purposes.

The detector background simulation predictions for CESR design conditions (600 mA total current; flat 3.5 nTorr pressure profile; $40 \%$ CESR duty factor; $15 \mathrm{keV} \mathrm{SR}$ threshold) are summarized in Table 2.

\begin{tabular}{c|c|c} 
Source & $\begin{array}{c}\text { SVX layer 1 Occ. } \\
\% \text { hits/layer/ } \mu \mathrm{s}\end{array}$ & $\begin{array}{c}\text { Dose } \\
\mathrm{krad} / \mathrm{year}\end{array}$ \\
\hline SR (tipscattering) & 0.007 & 0.1 \\
(backscattering) & 0.001 & 0.6 \\
Beam-Gas & 0.18 & 8 \\
\hline Total & 0.2 & 9
\end{tabular}

Table 2: Detector background simulation predictions for CESR design conditions

\subsection{Retractable Radiation Shields}

During CESR injection, the beams are horizontally separated at the interction point, making one of the beams further off-axis in the horizontally focusing IR quadrupole. As a result, large SR fluxes can be generated. Simulations show that SR doesn't directly strike the beampipe, but for added safety, retractable $\mathrm{W}$ shields were mounted on the beampipe [9]. The shields are constructed from $\mathrm{W}$ foil and have total thickness $200 \mu \mathrm{m}$. They are remotely actuated, and were designed to close during CESR injection and machine studies.

\subsection{IR Vacuum System}

As part of the Phase II interaction region upgrade new vacuum system components between $\pm 12 \mathrm{~m}$ from the IP were required. IR Pumping [8] is accomplished in large plenums incorporating massive titanium sublimation pumping (located at $\pm 2 \mathrm{~m}, \pm 6 \mathrm{~m}$ and $\pm 10 \mathrm{~m}$ from the IP).

\section{COMMISSIONING AND OPERATIONAL EXPERIENCE}

The IR components were assembled in early 1995, and the IR was installed from April-October 1995. CESR startup began October 29, 1995 and first $e^{+} e^{-}$collisions were obtained November 14. The SVX was turned on during collisions on November 21, 1995. Beam currents were initially limited by detector background levels for the first 2-3 months of operation as the storage ring pressure improved from beam processing. Since this initial start-up period, CESR currents have no longer been limited by detector backgrounds.

Figure 2 shows the dynamic pressure rise $(d P / d I)$ vs. accumulated beam dose measured at the $6 \mathrm{~m}$ TiSP chambers, demonstrating the effects of beam processing (and TiSP flashing) since Phase II start-up. For orientation, after the first month $\sim 10 \mathrm{~A}-\mathrm{hr}$ had been accumulated, and after five months, $\sim 100$ A-hr had been accumulated. Now, after $\sim 700 \mathrm{~A}-\mathrm{hr}$, typical dynamic pressure rises in the IR are $\sim 0.002$ nTorr $/ \mathrm{mA}$ [8].

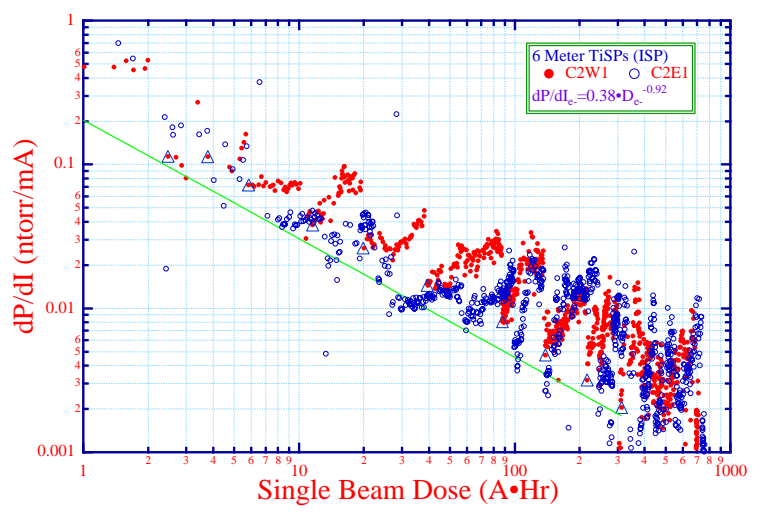

Figure 2: Dynamic pressure rise vs. beam dose for the $6 \mathrm{~m}$ TiSP chambers since CESR Phase II start-up.

\subsection{Radiation Levels}

The IR radiation history since Phase II start-up is shown in Figure 3. Nearly $2 \mathrm{krad}$ was logged during the initial 2 month beam processing phase. About $10 \mathrm{krad}$ have been logged since start-up, about half of which was accumulated with the SVX electronics powered. Since CESR beam currents have been nearly constant for the past $\sim 6$ months, the radiation has been increasing linearly with time, at a rate of $\sim 8 \mathrm{krad} /$ year at peak total currents of $\sim 320 \mathrm{~mA}(\sim 4.6$ $\mathrm{krad} /$ year powered). The accumulated powered dose represents about $20 \%$ of the lifetime dose for the SVX readout electronics.

Of the total accumulated dose since Phase II start-up, $63 \%$ was delivered during high energy physics data-taking, $17 \%$ during CESR Injection, and $20 \%$ during other activities, including machine studies.

\subsection{Beampipe Cooling}

The beam-generated heat load on the central Be pipe has been measured [10] by reducing the coolant flow and storing large beam currents, noting the temperature rise. 15$20 \mathrm{~W}$ was measured at $300 \mathrm{~mA}$ total current ( 36 bunches and $\sim 8.3 \mathrm{~mA} /$ bunch). The heat load has also been measured 


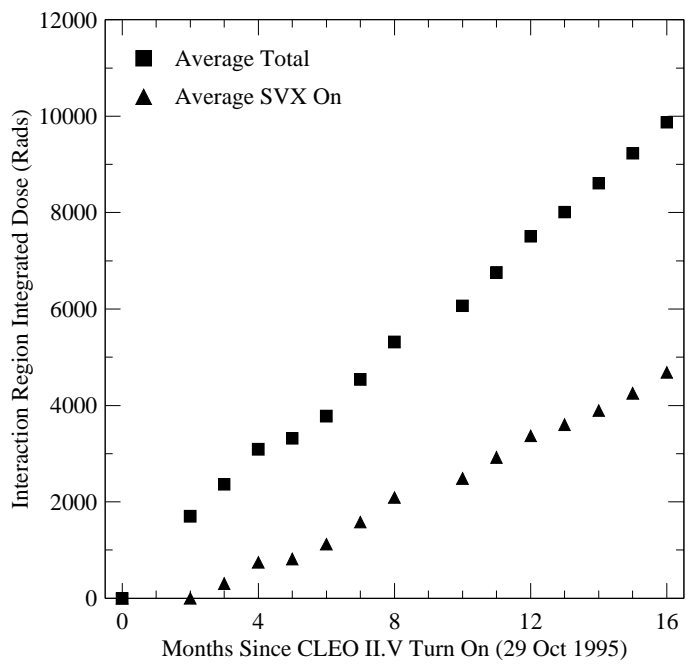

Figure 3: IR radiation history since CESR Phase II start-up.

by noting the temperature rise during data-taking periods, although the heat generated by the nearby SVX detector electronics $(\sim 200 \mathrm{~W})$ is a much larger source, making extraction of the small beam-related power difficult. Scaling this heat load to the design current of $600 \mathrm{~mA}$ gives at most $80 \mathrm{~W}$, well within the design capacity of $400 \mathrm{~W}$.

The measured heat loads are consistent with calculated values, given the uncertainties involved. The expected image current heating is $\sim 3 \mathrm{~W}$; the HOM power generated in the IR mask structure is $\sim 6 \mathrm{~W}$ at these beam currents.

\section{COMPARISON OF BACKGROUND WITH SIMULATION}

A comparison of measured background rates with detector background simulation predictions has been undertaken. Two simulation tools are in use at CESR, one for beam-gas backgrounds and another for SR backgrounds [11]. Each begins with the CESR lattice description, vacuum chamber geometry, and closed orbit and interaction region bump strengths. The beam-gas simulation generates Coulomb and bremsstrahlung interactions throughout the machine and tracks the secondaries, collecting those beam particles and photons that strike near the IP. These particles are used as input to a GEANT-based Monte-Carlo simulation of the detector response. The SR simulation generates photon fluxes from a 2-D description of the beam, and follows their trajectories to vacuum chamber surfaces where fluxes are accumulated. These calculated fluxes are used as input to an $x$-ray scattering Monte-Carlo simulation, which propagates scattered photons (from Compton scattering, Rayleigh scattering and fluorescence) to the detector where the response is simulated.

\subsection{Single Beam Results}

We have compared single-beam radiation levels, measured with the beampipe radiation monitoring system, with simulation predictions. For a single beam of $140 \mathrm{~mA}$, the average IR radiation was $0.8 \mathrm{rads} / \mathrm{hr}$, compared with a simulation prediction (including the measured pressure profile and IR bumps) of 0.75 rads/hour ( 0.25 from SR, 0.50 from beam-gas). Although the agreement for this set of conditions is quite good, other results revealed discrepancies. Namely, the simulation failed to predict the dependence of background rates on the horizontal displacement bump, an IR bump (used to displace the collision point) which is routinely used to optimize background levels.

\subsection{Pressure Bump Studies}

Preliminary pressure bump studies also revealed disagreement. By introducing calibrated leaks and turning off pumps in different sectors of the machine, the "sourceeffectiveness" (the contribution of a single source point to detector backgrounds) of different portions of the ring was measured. We observed that only the region from $0-30 \mathrm{~m}$ from the IP contributes to detector backgrounds; the IP is "masked" from lost-particles generated elsewhere. (This result is predicted by simulation.) In addition, we observed that $\sim 2 / 3$ of the background is generated within $12 \mathrm{~m}$ from the IP (the IR straight and the nearest dipole) and $\sim 1 / 3$ is generated in the "hard-bend" region (the region 14-40m from the IP which contains stronger bends), a result for which the beam-gas simulation failed to account.

In order to better understand the "hard-bend" contribution to detector backgrounds, a calibrated CO leak was introduced at $\sim 15 \mathrm{~m}$ from the IP [12]. Different pressure profiles were generated (shown in Figure 4a) by systematically turning off distributed and lumped pumps one-by-one in the hard bend region to obtain a set of 7 pressure conditions. Pressure profiles were calculated (based on available pressure measurements) with a one-dimensional finite element method in which the pumping speed of each pump and thermal outgassing rate are adjusted to obtain the best fit. For each condition, IR radiation levels were measured with a small stored beam current. The IR radiation response for each of the 7 cases, normalized to beam current, is shown in Figure 5 (curve "rad_meas").

Since the beam-gas simulation did not properly account for the hard-bend contribution, another simpler approach was taken. The source-effectiveness for IR mask strikes was calculated as follows. At each source location, the range of energy losses which produce trajectories that strike the IR mask (without hitting an aperture between the source point and mask) is calculated. A bremssstrahlung weight is assigned to that location from the bremsstrahlung cross-section integrated over the range of calculated energy-losses. Likewise, a Coulomb weight is calculated by determining the range of scattering angles which lead to trajectories that strike the IR mask. The total weight (bremssstrahlung and Coulomb) is plot- 
ted as a function of the source location in Figure $4 \mathrm{~b}$ (curve "effect_SDH_tot_p2"; relative strength shown on the right axis). This calculated source effectiveness clearly shows two contributions: one lobe from $0-10 \mathrm{~m}$, and another from $16-27 \mathrm{~m}$.

The calculated source-effectiveness is compared to the measured radiation rates for various pressure profiles, up to a normalization factor, by multiplying the pressure profile times the source-effectiveness. The overall normalization is obtained by choosing two pressure profiles (P2-P3) whose difference corresponds to a pressure bump near $20 \mathrm{~m}$ (shown in Figure 4b, curve "P2_minusP3") which overlaps the predicted source effectiveness. The pressure bump times the effectiveness divided by the measured difference in radiation between the two cases provides the normalization factor. The calculated radiation response for the 7 cases, using this normalization factor, is displayed in Figure 5 (curve "RAD_cal_norm"). The agreement between the measured radiation response and the calculated response is excellent.

These results confirm that there is a significant contribution to backgrounds from the "hard-bend" region, a result explained by a simple calculation of lost particle trajectories and fluxes. Work is underway to reconcile the simple source-effectiveness calculation described here with the full Monte-Carlo simulation.
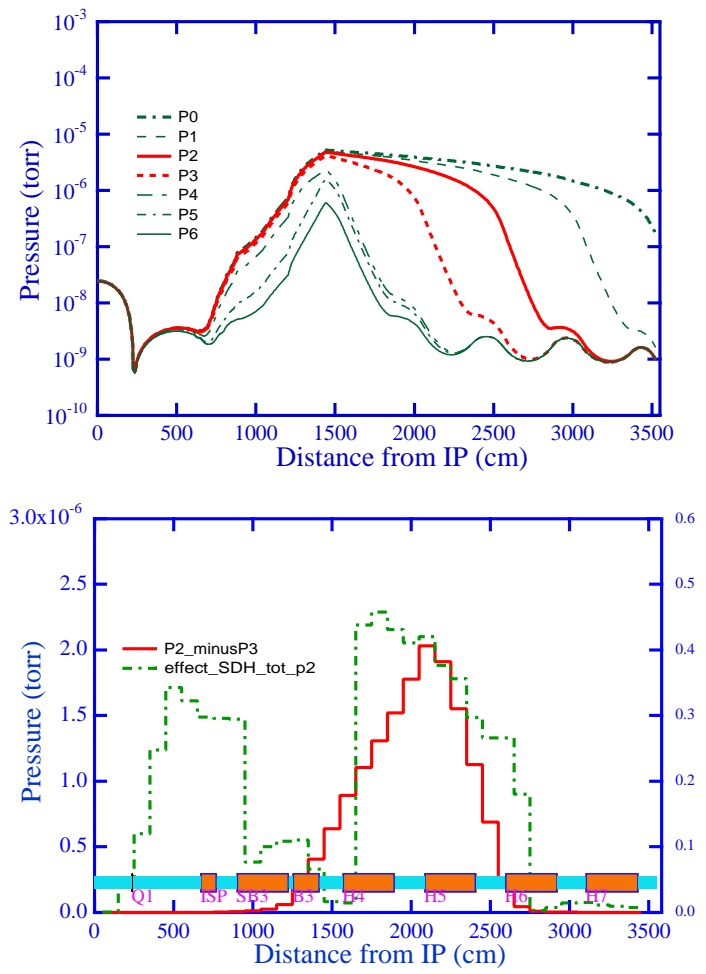

Figure 4: (Top) Pressure profiles for a calibrated CO leak experiment. The leak was introduced at $15 \mathrm{~m}$. (Bottom) The calculated source-effectiveness for IR mask strikes is shown (right axis) together with a pressure bump resulting from the difference in profiles $\mathrm{P} 2$ and $\mathrm{P} 3$.

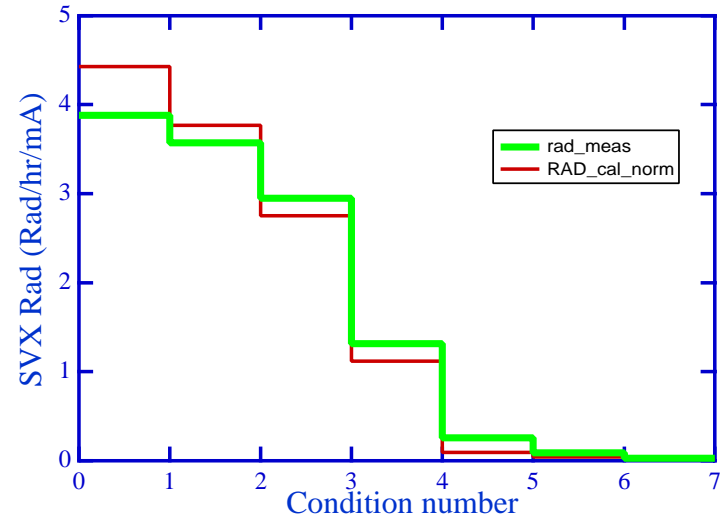

Figure 5: Measured IR radiation response is compared with the calculated response for the 7 pressure profiles shown in Figure 4.

\section{CONCLUSIONS}

The design and performance of the CESR Phase II Interaction Region has been reviewed. The IR shielding performance is as expected, although detailed features of the background sources are still under investigation. Meanwhile, CESR luminosity continues to improve, having delivered $3.9 \mathrm{fb}^{-1}$ since Phase II start-up [2].

\section{ACKNOWLEDGEMENTS}

The author would like to thank D. Cinabro, Y. Li and N. Mistry for results presented in this paper.

\section{REFERENCES}

[1] D. Cinabro, "A Silicon Vertex Detector for CLEO," Proc. XXVI ICHEP (Dallas, TX, 1992) 1727.

[2] D. Rubin, "CESR Status," these proceedings.

[3] R. Meller, Cornell Note CON-90-17, 1990.

[4] J.P. Alexander et. al., NIM A337 (1993) 171.

[5] Stuart Henderson, "The CLEO-II SVX Water-cooled Be Beampipe," Proc. 8th Meeting of DPF (Albuquerque, NM), World Scientific, (1994) 1480.

[6] Stuart Henderson and Scott Roberts, Cornell CBX-97-15.

[7] S. Henderson, "The CESR-CLEO Interaction Region Upgrade,” Proc. XXVI ICHEP (Dallas, TX, 1992) 2022.

[8] N. Mistry, "Massive Titanium Sublimation Pumping in the CESR Interaction Region", these proceedings.

[9] D. Dumas, Cornell CBX-95-106.

[10] D. Cinabro, private communication.

[11] “CESR-B Conceptual Design Report," (1993)

[12] N. Mistry, Cornell CON-97-02. 